\title{
Oral Immunotherapy for the Treatment of Chronic Diseases and Infections
}

\author{
George J Georgiou* \\ Research Director, Worldwide Health Center, Cyprus.
}

*Corresponding author: George J Georgiou, Research Director, Worldwide Health

Center, Cyprus E-mail: admin@worldwidehealthcenter.net.

Received Date: September 05, 2018

Published Date: September 19, 2019

\section{Abstract}

Immunotherapy has recently emerged as a novel and appealing strategy for treating cancer and other acute and chronic diseases. During an infection, macrophage phagocytic cells proliferate in order to counteract the infection.

For the last 20 years, the group-specific component (Gc) protein-derived Macrophage Activating Factor (GcMAF), has received a lot of attention. In 1999, the Japanese researcher Nobuto Yamamoto published his first report mentioning the use of Gc -MAF on Tumour Bearing Mice, along with another group the same year. It has received a great deal of attention in the past few years because of its proposed therapeutic use in the immunotherapy of cancer and other diseases ranging from autism and AIDS to multiple sclerosis and lupus.

There is substantial scientific evidence in the last 20 years, demonstrating that GcMAF, extracted from human blood, inhibits cancer cell proliferation in vitro, angiogenesis and tumour growth in the experimental animal, and may have a role in the immunotherapy of a variety of conditions. [1-10].

Further research has shown that blood derived GcMAF may also play a significant role in reducing the damage inflicted by chemotherapy in neurons and glial cells in vitro [11,12]. This finding will provide an excellent protective factor for those doing chemotherapy.

There is an alternative way of producing GcMAF by taking oral colostrum macrophage-activating factor (MAF) produced from bovine colostrum. This has shown high macrophage phagocytic activity as well, is easy to dissolve and drink and is not as expensive and difficult to use as the human blood derived GcMAF which involves injecting.

This paper discusses the science behind such an oral formulation.

The aim of this review is to provide insight into the natural components in these oral formulations responsible for immune modulation and some of the mechanisms involved. These new oral formulations, derived from buffalo colostrum, we have called Oral Colostrum Macrophage Activating Factor (OCMAF) or simply Colostrum MAF.

Oral Colostrum MAF has other advantages over injectable GcMAF - less chance of contamination from human blood serum and does not need to be injected by a doctor. Colostrum MAF is derived from bovine colostrum, a food source, and is considered a food or dietary supplement.

Additionally, another important benefit of oral colostrum MAF is that it did not mediate production of inflammatory cytokines, including; tumour necrosis factor cytokine interleukin-1s facto $\beta$ [13]. If colostrum MAF can be used to suppress the production of inflammatory cytokines, it can be an effective treatment for autoimmune diseases as well.

\section{Introduction}

Macrophages play an important role in the innate immune system that increase in numbers in response to an infection. They are phagocytic cells that recognize, engulf and destroy pathogens, cancer cells and foreign substances, release cytokines and are involved in the removal of cellular debris and clearing of cells that have undergone apoptosis [14].

Macrophages broadly consist of two classes: tissue-resident macrophages and infiltrating macrophages. Most tissues in the body contain tissue-resident macrophage populations [15]. All these macrophages, by definition, reside in their respective tissues and perform homeostatic tissue-specific functions [16-18].

Inflammatory monocytes, the source of infiltrating macrophages, selectively travel to the sites of inflammation, produce inflammatory cytokines and contribute to local and systemic inflammation [19]. Colostrum MAF has the added advantage of preventing the production of these inflammatory cytokines.

The general purpose of oral colostrum MAF immunotherapy is to optimize the health of the patient by improving quality of life, 
allowing the person to lead an active life, increase the chances of survival, optimize the effect of other therapies, regenerate the immune system, increase the number of monocytes and activate them to destroy cancer cells, viruses, bacteria and other pathogens in the body and, finally, increase the rate of maturation of dendritic cells [20].

As one of the major components of the oral colostrum formula is bovine colostrum, let us examine in some detail the components and physiological, biochemical and molecular functions of bovine colostrum.

\section{What Is Colostrum}

Colostrum is $50 \%$ or more protein, and rich in fat, vitamins and minerals. It is also the most zoo nutrient-rich of all foods, providing immune protein sub-fractions and peptides, growth factors and other lesser known zoonutrients (beyond the usual macronutrients such as immunoglobulins, bio-active peptides, enzymes, glycolconjugates and special amino acid bonds called double cystine).

The fats in colostrum provide essential fatty acids for cell development. Colostrum is also rich in sphingomyelin, cephalin, phosphatidyl choline and phosphatidyl serine that assist in development of the mucosal barrier, liver function, brain function, and/or immune function.

The vitamins include thiamin, riboflavin, pantothenic acid, pyroxidine, folic acid, vitamins E, C, B12, beta carotene and retinoic acids. Riboflavin is by far the richest in concentration, followed by folic acid. The minerals include calcium, chromium, iron, magnesium, phosphorus, potassium, sodium and zinc, with calcium being the most abundant.

\section{There are over 90 described components in bovine colostrum, that include:}

Cytokines: Small proteins produced by various cells in the body that induce the generation of specialized types of white blood cells, signal them to come to the site of an insult and help in their passage through tissues.

Cyclic adenosine monophosphate (cAMP): A phosphorylated nucleotide in a very specialized form that transfers the chemical energy necessary to drive metabolic reactions to form new protein, carbohydrate and fat molecules.

Enzyme inhibitors: These small proteins slow down or inhibit the breakdown of proteins by certain enzymes. They provide limited protection to the immune, growth and metabolic factors as they pass through the digestive tract.

Fat-associated vitamins: Significant quantities of vitamins A, $\mathrm{D}, \mathrm{E}$ and $\mathrm{K}$ are dissolved in or associated with the fat in colostrum.

Gut Protective Substances: Immunoglobulins (IgG, IgM, IgA). Complex proteins, better known as antibodies, that make up a significant portion of the proteins found in complete first milking colostrum. These antibodies offer protection against bacteria, viruses and fungi that infect the gastrointestinal tract of humans.
Immuno-regulating Substances: Thymosin (alpha \& beta chains). A hormone composed of two protein-based chains that are present in bovine colostrum that acts on the thymus gland to stimulate activation, development and maintenance of the immune system.

Insulin: A hormone required for the effective utilization of glucose (blood sugar) in the body. Insulin binds to specific sites on cells, facilitating their interaction with IGF-1 and, thus, initiating the conversion of glucose to glycogen, a high energy source carbohydrate.

Lactoferrin: A mineral-binding carrier protein that attaches to available iron. Certain aerobic bacteria, like E. coli, require iron to reproduce and, therefore, lactoferrin is an effective substance, when operating in the presence of a specific antibody, to impede the growth of some microorganisms in the gut.

Lactoperoxidase: A mildly effective enzyme that can also attach to the wall of certain bacteria, degrade selected proteins and interfere with the ability of the bacteria to replicate themselves.

Lymphokines: Different sized proteins produced by different types of white blood cells that tell related cells to transform themselves into more functional cell types that can release substances capable of destroying an invading microorganism.

Lysozyme: A very powerful enzyme that can attach itself to the cell wall of certain pathogenic bacteria and degrading select components, leaving holes in the wall of the bacteria.

Mineral-binding proteins: The iron-binding proteins, lactoferrin and transferrin, have already been discussed above. In addition to interfering with the replication of certain microorganisms, they also serve to capture iron from the ingested food and present it in a form that can readily be absorbed by the body. Lactoferrin can also bind copper and deliver it in a form suitable for absorption by the body. In addition, there are two carrier proteins in colostrum that assist in the absorption of calcium. They are casein, which is also an abundant source of amino acids to build new protein molecules, and alpha-lactalbumin, which is present in colostrum very soon after birth.

Oligosaccharides and glycoconjugates: Complex carbohydrates (sugars) that can adhere to specific sites on the inner surface of the gastrointestinal tract and prevent the attachment of microorganisms.

Proline Rich Polypeptides (PRPs): Colostrum is rich in bio-active immune modulating peptides variously known as infopeptides, cytokine precursors, colostrinin, colostrinine, transfer factor and proline rich polypeptides (PRP). PRPs are short chains of amino acids with a high concentration of the amino acid proline, called polypeptides, with a molecular weight of 6000 Daltons. They support the regulation of the thymus, the gland responsible for the normal development of immunologic function in the body [21-23]. Proline rich polypeptides promote proper response to microbial invaders, toxins or allergens through cytokine modulation and 
natural killer cell (NK) activity [22-26]. Colostrum active peptides (PRPs) also promote thymopoeisis of naïve T Cells and modulate Th2, generally promoting a "shift to the left", down regulating Th2 and up regulating Th1, if these are out of balance [27].

Transfer factors: Small proteins produced in response to the body's exposure to certain types of microorganisms, particularly those that reside in deep tissues for a long period of time, like the bacterium that causes tuberculosis. They are specific for a particular microorganism and are carried inside of certain types of specialized white blood cells.

Transferrin: Another mineral-binding carrier protein that attaches to available iron and can act independently or in concert with lactoferrin to impede the growth of certain aerobic bacteria, particularly in the gut.

Xanthine Oxidase: Another mildly effective enzyme that can also attach to the wall of certain bacteria, degrade different proteins than those affected by lactoperoxidase and, therefore, also interfere with the ability of the bacteria to replicate themselves.

Vitamin-binding proteins: Smaller proteins that act as carriers to deliver B-complex vitamins to the body. Carrier proteins and the associated vitamins folate (B6), B12 and orotic acid are found in colostrum.

White blood cells (leukocytes): Primarily, three types of functional white blood cells are present in colostrum, including neutrophils, macrophages and polymorphonuclear cells. Each can phagocytize (engulf) microorganisms and other foreign bodies and apply substances carried internally to the destruction of the microorganisms. Their functions are dramatically enhanced when antibodies first attach to the microorganisms.

In addition to these important ingredients, there are also other growth factors and metabolic factors that play critical roles in the immune system:

The Growth Factors: Insulin-like growth factors (IGFs): Insulinlike growth factor-1 (IGF-1) and its closely related counterpart insulin-like growth factor-2 (IGF-2) are potent hormones that are found in association with almost all cells in the body. They are part of a group of more than 90 different proteins, called the "IGF Binding Protein (IGFBP) Superfamily", that is responsible for the processes by which cells grow and reproduce. These substances are also responsible for maintenance of the metabolic pathways in the body by which cells convert glucose to glycogen, a primary energy resource, and use amino acids to create proteins. The key event that triggers the functions of the various proteins in the IGFBP Superfamily is the attachment of IGF-1 to a specific site on the surface of a cell. Many of the growth factors found in colostrum and previously defined by their functions are now considered part of the IGFBP Superfamily. This includes the following substances, among others.

Transforming growth factors, A \& B: Induces the transformation of cells from an immature form to a mature, functional status.
Epithelial growth factor: Involved in the generation and maintenance of cells in the epithelial (outer) layers of the skin.

Fibroblast growth factor: Associated with the regeneration of various types of tissue, including skin and other organs.

Platelet-derived growth factor: Responsible for the generation of cells and functions associated with blood clotting.

The Metabolic Factors: Leptin: A small hormone-like protein that can suppress appetite and lead to body weight reduction. Mature fat cells (adipocytes) release leptin in the presence of insulin, which is also found in colostrum. Insulin-producing pancreatic beta-cells have binding sites for leptin and it is believed that the size of fat cells may be a major factor in determining the amount of leptin released. Therefore, leptin deficiency may be associated with obesity, particularly in diabetic individuals.

Now that we have looked at the components of colostrum, let us examine one of the important Macrophage Activating Factors that are formed in the body with the help of the colostrum.

\section{What Is GcMAF}

GcMAF or Gc protein-derived macrophage activating factor is a protein produced by modification of vitamin D-binding protein. Vitamin D-binding protein belongs to the albumin gene family, together with human serum albumin and alpha-fetoprotein. It is a multifunctional protein found in plasma, ascitic fluid, cerebrospinal fluid and on the surface of many cell types.

Human GC is a glycosylated alpha-globulin, $\sim 58 \mathrm{kDa}$ in size. Its 458 amino acids are coded for by 1690 nucleotides on chromosome 4 (4q11-q13). The primary structure contains 28 cysteine residues forming multiple disulphide bonds. Gc contains 3 domains. Domain 1 is composed of 10 alpha helices, domain 2 of 9 , and domain 3 of 4 [28].

It can bind the various forms of vitamin D including ergocalciferol (vitamin D2) and cholecalciferol (vitamin D3), the 25-hydroxylated forms (calciferol), and the active hormonal product, 1,25-dihydroxyvitamin D calcitriol. The major proportion of vitamin D in blood is bound to this protein. It transports vitamin D metabolites between skin, liver and kidney, and then on to the various target tissues [29].

Biochemically, GcMAF results from sequential deglycosylation of the vitamin-D binding protein (the Gc protein), which is naturally promoted by lymphocytes (B and $\mathrm{T}$ cells). The resulting protein may be a macrophage activating factor (MAF) [30]. MAFs are lymphokines that control the expression of antigens on the surface of macrophages, and one of their functions is to make macrophages become cytotoxic to tumours [31].

Yamamoto and others propose that the transformation of Gc protein to GcMAF in vivo comes about owing to an inflammationtriggered increase in the expression of beta-galactosidase and sialidase on the surfaces of $\mathrm{B}$ and $\mathrm{T}$ lymphocytes, respectively; GcMAF can be produced in vitro by exposing Gc protein to immobilized beta-galactosidase and sialidase [32 - 43] (Figure 1). 


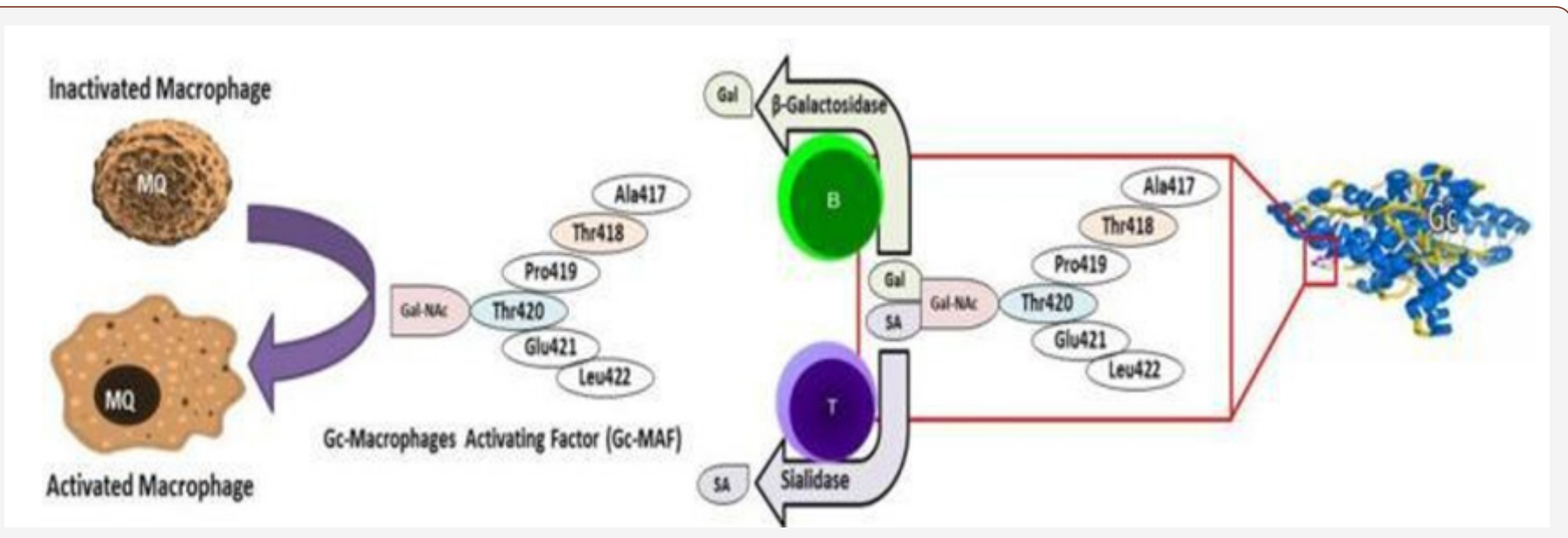

Figure 1: Gc-MAF generation cascade.

Gc: Group specific component; Gal: Galactose NAc: N-Acetyl SA; Sialic Acid T: T-Cell Lymphocyte B: B- Cell Lymphocyte

The natural concentration of GcMAF in the blood of humans is approximately $4 \mathrm{mg} / \mathrm{L}$.

\section{The Research and the Controversy around GC- Globulin and GcMAF}

We have already examined the encouraging evidence for the use of GcMAF, both serum and oral based. However, the research is showing considerable inconsistencies at the molecular and clinical level that force us to question the interpretation of the results reported above [44].

One such inconsistency that contradicts some of the published research concerns the cancer risk in individuals harbouring the Gc2 allele only (Gc2 homozygotes) of the Gc protein. These individuals are unable to glycosylate the Gc protein on Threonine 420 due to its substitution by lysine. Thus, there is no GalNAc in position 420 .

This basically means that Gc2 homozygotes are unable to produce a single molecule of GcMAF but, despite this fact, the risk of cancer, particularly breast cancer in these individuals is decreased rather than increased as one would have expected given the absence of bona fide GcMAF [45-46].

So, these findings are contradictory to the role of GalNAc in position 420 of the Gc protein and immune competency and cancer risk. In addition, there is other research that has shown that Nagalase ( $\alpha$-N-acetylgalactosaminidase), an enzyme produced by cancer cells and viruses that would remove GalNAc and prevent the conversion of of Gc protein to GcMAF [36]. It appears, however, that elevated Nagalase does not necessary deplete blood levels of GcMAF.

Another significant inconsistency found in research refers to the amount of endogenous GcMAF that, at variance with what had been hypothesized, is not decreased in cancer patients, and is actually much higher than the amount of exogenous GcMAF that has been administered in the immunotherapy of cancer as demonstrated by [36].

Rehder and his research team examined the glycosylation status of the Gc protein in 56 patients with breast, colorectal, pancreatic, and prostate cancer (i.e. those cancers that had been successfully treated with GcMAF), and observed that there was no significant depletion of GcMAF in the 56 cancer patients examined relative to healthy controls. The question raised here is if indeed these patients were treated using GcMAF, how could this have occurred?

Such an inconsistency concerning the role of nagalase, is further corroborated by the observation that autistic children may show levels of serum nagalase higher than those of HIV or cancer patients but, nevertheless, show no signs of immunodeficiency [47-50].

There is another interesting conclusion from various research studies that shows Gc protein binding to chondroitin sulphate, a plasma glycosaminoglycan, something that can occur in serum colostrum which contains relatively high amounts of both the Gc protein as well as chondroitin sulphate [51-53].

Interestingly, chondroitin sulphate favours the intracellular delivery of arginine-rich peptide-DNA complexes by coating the surface of these complexes through electrostatic interactions which improves their extracellular stability and subsequent cellular entry [54].

Based on these interesting observations, it is clear that the addition of chondroitin sulphate to an oral colostrum formulation would have many benefits given that the the Gc protein, whether deglycosylated or not, binds chondroitin sulphate both on the cell surface and in bodily fluids, and the resulting multimolecular complexes, under the form of oligomers trigger a transmembrane signal or, alternatively, are internalized and convey the signal directly to the nucleus thus eliciting the diverse biological effects observed for both GcMAF and chondroitin sulphate.

\section{The Development of Oral Colostrum}

Given the powerful immune modulating properties of bovine colostrum, with its macrophage activating factors, various researchers began to look at producing oral colostrum as opposed to serum GcMAF which was used to activate macrophage phagocytosis. This oral colostrum was shown to have similar activities to the injectable GcMAF [55]. Thus, colostrum MAF, a new form of a macrophage activating factor, has been developed. 
In order for the body to convert and utilize the oral colostrum proteins and other factors to GcMAF in the body, many other components are required, such as vitamin $\mathrm{D}$, oleic acid, chondroitin sulphate A, B and C, conjugated linoleic acid (CLA), as well as albumins from bioactive eggs rich in IgY.

Many of the commercial oral colostrum products being sold today for activating macrophage phagocytosis are deficient in most of these important factors that we will discuss below. If the body is deficient in these other factors, the chances are that the oral colostrum will not be utilised by the body in the correct way, therefore greatly reducing its macrophage phagocytotic activities.

Let us examine the biochemical and physiological significance of these other components in oral colostrum formulas.

\section{The Role of Vitamin D and Immune Regulation}

Based on these research findings stated above, it is clear that Vitamin D is critical to the total health of the human body and defends the immune system from an attack by chronic disease. Researchers continue to underestimate the role that Vitamin D plays in supporting biological activities. This powerful nutrient has only recently been analysed for its role in influencing GcMAF availability and function.

Without Vitamin D, GcMAF cannot be produced. GcMAF contains binding proteins and cellular receptors requiring vitamin D activation. Vitamin D3 must be converted into GcMAF by a key biological reaction in the body. A carbohydrate is bonded with Vitamin D3 through a process called glycosylation. GcMAF then activates macrophages which act as the body's surveillance team against destructive agents and infection.

\section{The Role of Chondroitin Sulphate in Immunity}

We have already seen how chondroitin sulphate binds to Gc protein in its immune regulating functions. In 1999, DiMartino and Kew studied the interaction of the Gc protein with the cellular membrane of neutrophils and demonstrated that the Gc protein did not bind to a specific cellular receptor but formed oligomers and interacted with a chondroitin sulphate proteoglycan on the cell surface [56].

According to these researchers. large heterogenous macromolecules, such as cell surface proteoglycans containing chondroitin sulphate, would explain the unusual cell-binding characteristics of the Gc protein. Interestingly, chondroitin sulphate is composed of a chain of alternating sugars that are GalNAc and glucuronic acid.

It appears that several molecules of Gc protein could be ssembled as oligomers as they bind to the chondroitin sulphate that is present in the extracellular matrix surrounding the cellular membrane. It is possible, based on this molecular model, that the assembly of oligomers of Gc protein may trigger a transmembrane cell signaling mechanism.

The inconsistencies in the GcMAF research may be explained by the formation of such a multimolecular complex comprising oligomers of the Gc protein and chondroitin sulphate, that is inherently rich in GalNAc.

Interestingly, chondroitin sulphate shows all the biological and clinical features that have been attributed to GcMAF - it activates macrophages and induces the synthesis and release of nitric oxide in a manner superimposable to that described for GcMAF $[57,58]$.

In analogy with the reported effects of GcMAF, activation of macrophages by chondroitin sulphate is not accompanied by the release of pro-inflammatory cytokines or Prostaglandin E2 Ruggiero M, et al [57], further highlighting its role as an immune modulator with no pro-inflammatory activity.

Experimental and clinical data suggest that chondroitin sulphate might be a useful therapeutic agent in diseases as diverse as osteoarthritis, psoriasis, inflammatory bowel diseases, atherosclerosis, Parkinson's and Alzheimer's diseases, multiple sclerosis, amyotrophic lateral sclerosis, rheumatoid arthritis and systemic lupus erythematosus, i.e. many of the conditions that have been reported to respond to GcMAF treatment [59].

The Mayo Clinic of the United States of America, for example, states that there is strong scientific evidence for the clinical use of chondroitin sulphate in osteoarthritis. As well as in conditions as diverse as coronary artery disease, psoriasis, muscle soreness or interstitial cystitis. The website of the Mayo Clinic reports that there are clinical studies supporting its use, although further studies need to be performed.

However, most interesting is the list of the uses for chondroitin sulphate based on tradition or scientific theories reported by the Mayo Clinic. Such a list encompasses: “aging, allergies, Alzheimer's disease, amyotrophic lateral sclerosis, antioxidant, antiviral, blood clots, bone healing, breast cancer, burns, cervical disc disease, chest pain, chronic venous ulcers, clogged arteries, colorectal cancer, diabetes, gout, gum disease, headaches, heart attack prevention, heart disease prevention, HIV/AIDS, hyperglycaemia, high cholesterol, inflammation, inflammatory bowel disease, joint problems, kidney stones, leukaemia, lung cancer, malaria, mouth and throat infections, multiple sclerosis, nerve damage, nerve regeneration, neuroblastoma, osteoporosis, pain, Parkinson's disease, premature birth prevention, quality of life (osteoarthritis), rheumatoid arthritis, snoring, soft tissue injury, spinal cord injury, spine problems, surgery, systemic lupus erythematosus, temporomandibular joint disorder (TMJ), transplants, wound healing" [60].

Let us now examine some of the other components that would be beneficial to have in an oral colostrum formulation and the reasons why.

\section{Oleic Acid}

A group of researchers Branca JJV et al in 2015 found that combining GcMAF with Oleic Acid, a component of olive oil, activates human spleen macrophages which in turn phagocytise cancer cells. In addition, it was observed that GalNAc-DBP complexed with oleic acid activates human spleen macrophages in vivo as documented by colour-doppler ultrasonography of the spleen [61]. 
These results demonstrate that GalNAc-DBP complexed with oleic acid has a double effect on myeloma and Hodgkin's lymphoma cancer cells: a direct inhibition of their proliferation and viability and, at the same time, an efficient macrophage activation leading to a significant depletion of cancer cell population. Taken together, these results suggest that GalNAc-DBP complexed with oleic acid may prove effective in the integrative immunotherapy of multiple myeloma and Hodgkin's lymphoma [62].

It has been known since 1988 that oleic acid is non-covalently bound to the vitamin D binding protein (DBP) that constitutes one of its carrier proteins in plasma and other biological fluids [63].

The research has recently elucidated the molecular interactions between oleic acid, vitamin D, DBP and the vitamin D receptor [64]. In this context, it is worth noting that the anti-cancer effects of oleic acid are mediated by its interaction with proteins that are found in biological fluids such as milk or plasma [65]. Therefore, it can be hypothesized that DBP, by virtue of its complexing with oleic acid, may play a role in anti-cancer immune surveillance that goes beyond that of a carrier protein.

It has been proposed that the anticancer effects of $\mathrm{OA}$ are mediated by its interaction with proteins highly represented in biological fluids such as $\alpha$-lactalbumin and lactoferrins. These proteins bind $\mathrm{OA}$ to form OA-protein complexes which exhibit highly selective anti-tumour activity in vitro and in vivo [66].

Soon after their identification, these complexes were labelled as HAMLET an acronym that stands for "human $\alpha$-lactalbumin made lethal to tumour cells", even though further studies demonstrated that $\alpha$-lactalbumin is not the sole protein forming such complexes. Thus, other proteins, forming complexes with OA, exhibit identical anticancer properties $[46,66]$.
It is now accepted that these OA-protein complexes destroy tumour cells with high selectivity and with no evidence of toxicity for normal tissues, a key feature in the quest for anticancer treatments devoid of toxic side effects.

Study of the structural characteristics of such anticancer OAprotein complexes demonstrated that a common molecular feature is the tendency toward OA-induced protein oligomerization. Since OA-induced oligomerization has been reported for a number of proteins in addition to those initially identified in HAMLET, it was hypothesised that this phenomenon may be inherent to many proteins [66].

Avacado powder is a good way of adding the oleic acid to an oral colostrum formulation as the percentage of oleic acid in its total fatty acid content is greater than $50 \%$ and is probably the richest fruit in oleic acid.

\section{Conjugated Linoleic Acid}

Conjugated Linoleic Acid (CLA) refers to a group of polyunsaturated fatty acids that exist as positional and stereoisomers of octadecadienoate $(18: 2)[67,68]$. The double bonds of CLA may be in the positions of 7,9; 8,10; 9,11; 10,12; or 11,13 and the 3-dimensional geometric combinations of cis and/or transconfigurations.

Numerous physiologic properties have been attributed to CLA including action as an anticarcinogenic, antiatherosclerotic, antiadipogenic and antidiabetogenic agent $[69,70]$. In addition, CLA modulates immunity and thrombosis as well as fatty acid biochemistry, lipid metabolism and gene expression in the liver, muscle and adipose tissues [70]. There are several recent reviews on the effects and mechanisms of CLA in biological systems, including cancer [71].

\section{Discussion}

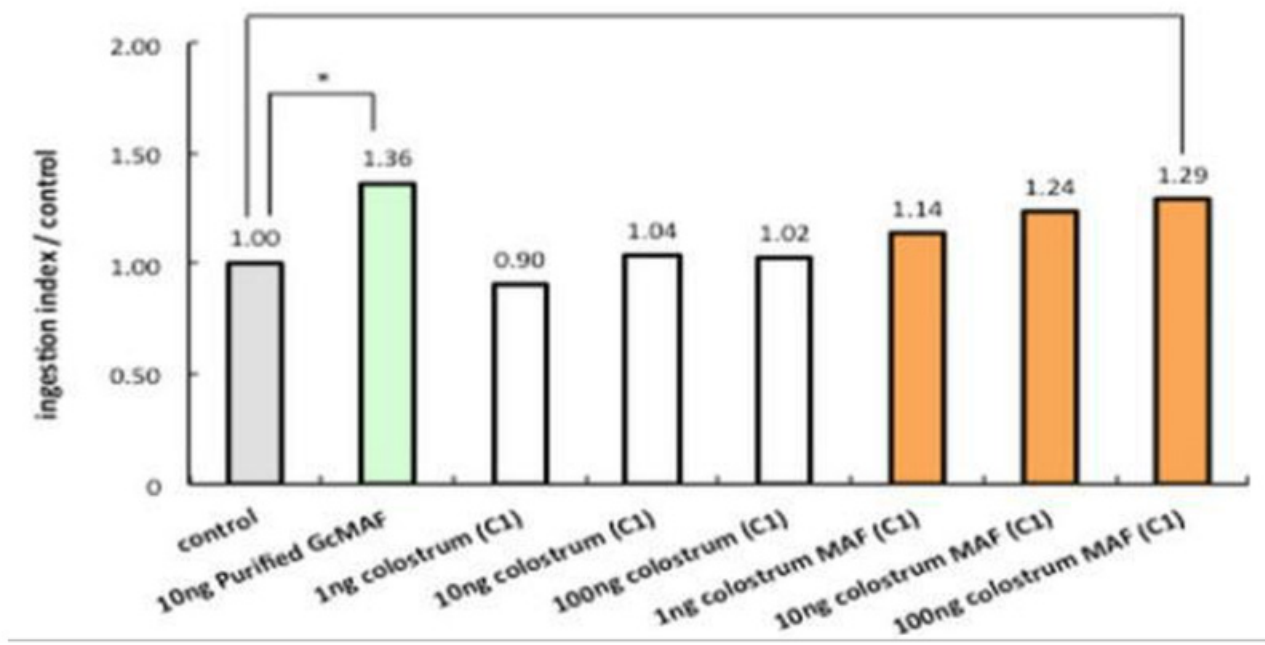

Figure 2: Phagocytic activity of oral colostrum GcMAF. (Researched by Saisei Mirai Research Centre, Japan).

A good oral colostrum formulation, based on what has been discussed in this paper, would therefore contain bovine colostrum from buffalo and cow's colostrum, oleic acid, vitamin D, chondroitin sulphate, IgY and lactoalbumins from eggs and conjugated linoleic acid. In nature the phenomenon of synergy is often encountered where the whole is not always the sum of the individual parts, and 
this is the case with such a formulation. This formulation could help modulate the immune system of people with acute and chronic infections and chronic diseases, including cancer. There are many disease processes that can benefit, as was previously listed by the Mayo Clinic. Regarding dosages, when comparing the Phagocytic activity of Colostrum GcMAF with that of the first-generation human serum based GcMAF, it was found that it requires 10 times more oral Colostrum GcMAF for the same phagocytic activation, compared to serum GcMAF.

So, using 10 times as much oral colostrum MAF will bring the same effect as the first-generation serum injectable GcMAF -10 grams of the powered oral GcMAF would be the equivalent of about $4 \mathrm{ng}$ of colostrum MAF - if this is taken three times daily, then the dosage would be equivalent to $12 \mathrm{ng}$ of GcMAF. Injectable GcMAF that is usually given at $10 \mathrm{ng}$ per dosage daily - (Fig 2) [72]. The price of the oral colostrum formulation is also a fraction of the cost and easily obtainable [73].

\section{Acknowledgements}

None.

\section{Conflict of Interest}

The Author is affiliated with the trading name "Worldwide Health Center", a private organization in Cyprus that formulates and manufactures natural food supplements, including an oral Colostrum formula called MAF365. The author has not received financial compensation for writing this article.

\section{References}

1. Yamamoto N, Naraparaju VR, Srinivasula SM (1995) Structural modification of serum vitamin D3-binding protein and immunosuppression in AIDS patients. AIDS Res Hum Retroviruses 11(11): 1373-1378.

2. Yamamoto N, Naraparaju VR, Moore M, Brent LH (1997) Deglycosylation of serum vitamin D3-binding protein byalpha- $\mathrm{N}$-acetylgalactosaminidase detected in the plasma of patients with systemic lupus erythematosus. Clin Immunol Immunopathol 82(3): 290-298.

3. Inui T, Makita K, Miura H (2014) Case report: a breast cancer patient treated with GcMAF, sonodynamic therapy and hormone therapy. Anticancer Res 34(8): 4589-4593.

4. Ruggiero M, Ward E, Smith R (2014) Oleic Acid, deglycosylated vitamin Dbinding protein, nitric oxide: a molecular triad made lethal to cancer. Anticancer Res 34(7): 3569-3578.

5. Thyer L, Ward E, Smith R (2013) GC protein-derived macrophageactivating factor decreases a-N-acetylgalactosaminidase levels in advanced cancer patients. Onco immunology 2(8): e25769.

6. Inui T, Kuchiike D, Kubo K (2013) Clinical experience of integrative cancer immunotherapy with GcMAF. Anticancer Res 33(7): 2917-2919.

7. Siniscalco D, Bradstreet JJ, Cirillo A, Antonucci N (2014) The in vitro GcMAF effects on endocannabinoid system transcriptionomics, receptor formation, and cell activity of autism-derived macrophages. J Neuroinflammation 11: 78

8. Thyer L, Ward E, Smith R (2013) Therapeutic effects of highly purified deglycosylated GcMAF in the immunotherapy of patients with chronic diseases. American Journal of Immunology 9(3): 78-84.

9. Ward E, Smith R, Branca JJV, Noakes D, Morucci G (2014) Clinical experience of cancer immunotherapy integrated with oleic acid complexed with deglycosylated vitamin D binding protein. American Journal of Immunology 10(1): 23-32.
10. Bradstreet JJ, Vogelaar E, Thyer L (2012) Initial observations of elevated alpha-Nacetylgalactosaminidase activity associated with autism and observed reductions from Gc protein-macropahe activating factor injections. Autism Insights 4: 31.

11. Morucci G, Branca JJ, Gulisano M (2015) Gc-protein-derived macrophage activating factor counteracts the neuronal damage induced by oxaliplatin. Anticancer Drugs 26(2): 197-209.

12. Branca JJ, Morucci G, Malentacchi F, Gelmini S, Ruggiero M, et al. (2015) Effects of oxaliplatin and oleic acid Gc-protein-derived macrophageactivating factor on murine and human microglia. J Neurosci Res 93(9): 1364-1377.

13. Uto Y, Kawai T, Sasaki T (2015) Degalactosylated/Desialylated Bovine Colostrum Induces Macrophage Phagocytic Activity Independently of Inflammatory Cytokine Production. Anticancer Res 35(8): 4487-4492.

14. Mosser DM, Edwards JP (2008) Exploring the full spectrum of macrophage activation. Nat Rev Immunol 8(12): 958-969.

15. Davies LC, Jenkins SJ, Allen JE, Taylor PR (2013) Tissue-resident macrophages. Nature Immunology 14: 986-995.

16. Murray PJ, Wynn TA (2011) Protective and pathogenic functions of macrophage subsets. Nat Rev Immunol 11(11): 723-737.

17. Hashimoto D (2013) Tissue-resident macrophages self-maintain locally throughout adult life with minimal contribution from circulating monocytes. Immunity 38(4): 792-804.

18. Yang J (2014) Monocyte and macrophage differentiation: circulation inflammatory monocyte as biomarker for inflammatory diseases. Biomark Res 2(1): 1.

19. Castellana D (2014) Macrophages contribute to the cyclic activation of adult hair follicle stem cells. PLoS Biol 12(12): e1002002.

20. Toshio, Inui (2015) Oral Colostrum Macrophage-activating Factor for Serious Infection and Chronic Fatigue Syndrome: Three Case Reports. Anticancer Res 35(8): 4545-4549.

21. Leszek J, Inglot AD, Janusz M, Lisowski J, Krukowska K, et al. (1999) Colostrinin: a proline-rich polypeptide (PRP) complex isolated from ovine colostrum for treatment of Alzheimer's disease. A double-blind, placebo-controlled study. Arch Immunol Ther Exp (Warsz) 47(6):377385.

22. Janusz M, Starościk K, Zimecki M, Wieczorek Z, Lisowski J (1981) Chemical and physical characterization of a proline-rich polypeptide from sheep colostrum. Biochem J 199(1): 9-15.

23. World Intellectual Property Organization (2007) Novel Immunologically Active Peptide Fragments of a Proline-Rich Polypeptide Isolated from Colostral Mammalian Fluids for Treatment of Viral and Non-Viral Diseases or Diseased Conditions.

24. Inglot AD, Janusz M, Lisowski J (1996) Colostrinine: a proline-rich polypeptide from ovine colostrum is a modest cytokine inducer in human leukocytes. Arch Immunol Ther Exp (Warsz) 44(4): 215-224.

25. Starościk K, Janusz M, Zimecki M, Wieczorek Z, Lisowski J (1983) Immunologically active nonapeptide fragment of a proline-rich polypeptide from ovine colostrum: amino acid sequence and immunoregulatory properties. Mol Immunol 20(12):1277-1282.

26. Wieczorek Z, Zimecki M, Janusz M, Staroscik K, Lisowski J (1979) Prolinerich polypeptide from ovine colostrum: its effect on skin permeability and on the immune response. Immunology 36(4): 875-881.

27. Anonymous (2008). T-Cell Lymphocyte, Natural Standard Monograph.

28. Verboven C, Rabijns A, De Maeyer M, Van Baelen H, Bouillon R, et al. (2002) A structural basis for the unique binding features of the human vitamin D-binding protein. Nat Struct Biol 9 (2): 131-136.

29. Norman AW (2008) From vitamin D to hormone D: fundamentals of the vitamin D endocrine system essential for good health. Am J Clin Nutr 88 (2): 491S-499S

30. Malik Suneil, Fu Lei, Juras David James, Karmali Mohamed, Wong Betty YL, et al. (2013) "Common variants of the vitamin D binding protein gene and adverse health outcomes". Crit Rev Clin Lab Sci 50(1): 1-22. 
31. Mosser David M (2003) "The many faces of macrophage activation". J Leukoc Biol 73(2): 209-212.

32. Yamamoto N, Kumashiro R (1993) Conversion of vitamin D3 binding protein (group-specific component) to a macrophage activating factor by the stepwise action of beta-galactosidase of B cells and sialidase of $\mathrm{T}$ cells. J Immunol 151(5): 2794-2802.

33. Naraparaju VR, Yamamoto N (1994) Roles of beta-galactosidase of B lymphocytes and sialidase of $\mathrm{T}$ lymphocytes in inflammation-primed activation of macrophages. Immunol Lett 43(3):143-148.

34. Ono T, Ichikawa H, Asami R, Yamamoto I (1995) Enzymatically modified GC globulin induces the translocation of FcgRI and FcgRII from intracellular storage compartments to the cell surface in murine peritoneal macrophage. Jpn J Inflammation (15): 293-299.

35. Toyohara Y, Hashitani S, Kishimoto H, Noguchi K, Yamamoto N (2011) Inhibitory effect of vitamin D-binding protein-derived macrophage activating factor on DMBA-induced hamster cheek pouch carcinogenesis and its derived carcinoma cell line. Oncol Lett 2(4):685- 691.

36. Yamamoto N, Naraparaju VR (1998) Structurally well-defined macrophage activating factor derived from vitamin D3-binding protein has a potent adjuvant activity for immunization. Immunol Cell Biol 76(3):237-244.

37. Rehder DS, Nelson RW, Borges CR (2009) Glycosylation status of vitamin D binding protein in cancer patients. Protein Sci 18(10):2036-2042.

38. Ravnsborg T, Olsen DT, Thysen AH, Christiansen M, Houen G, et al. (2010) The glycosylation and characterization of the candidate Gc macrophage activating factor Biochim Biophys Acta 1804(4):909-917.

39. Yamamoto N, Homma S (1991) Vitamin D3 binding protein (groupspecific component) is a precursor for the macrophage-activating signal factor from lysophosphatidylcholine-treated lymphocytes. Proc Natl Acad Sci U S A 88(19): 8539-8543.

40. Borges CR, Jarvis JW, Oran PE, Nelson RW (2008) Population studies of Vitamin D Binding Protein microheterogeneity by mass spectrometry lead to characterization of its genotype-dependent Oglycosylation patterns. J Proteome Res 7(9): 4143-4153.

41. Yamamoto N, Naraparaju VR, Asbell SO (1996) Deglycosylation of serum vitamin D3-binding protein leads to immunosuppression in cancer patients Cancer Res 56(12): 2827-2831.

42. Nagasawa H, Uto Y, Sasaki H (2005) Gc protein (vitamin D-binding protein): Gc genotyping and GcMAF precursor activity. Anticancer Res 25(6A): 3689-3695.

43. Speeckaert M, Huang G, Delanghe JR, Taes YE (2006) Biological and clinical aspects of the vitamin D binding protein (Gc-globulin) and its polymorphism. Clin Chim Acta 372(1-2): 33- 42.

44. Ruggiero M, Reinwald H, Pacini, S ((2016)) Is chondroitin sulfate responsible for the biological effects attributed to the GC protein-derived Macrophage Activating Factor (GcMAF)? Med Hypotheses 94: 126-131.

45. Abbas S, Linseisen J, Slanger T (2008) The Gc2 allele of the vitamin D binding protein is associated with a decreased postmenopausal breast cancer risk, independent of the vitamin D status. Cancer Epidemiol Biomarkers Prev 17(6): 1339-1343.

46. Pacini S, Punzi T, Morucci G, Gulisano M, Ruggiero M (2012) Effects of vitamin Dbinding protein-derived macrophage-activating factor on human breast cancer cells Anticancer Res 32(1):45-52.

47. Thyer L, Ward E, Smith R (2013) A novel role for a major component of the vitamin D axis: vitamin D binding protein-derived macrophage activating factor induces human breast cancer cell apoptosis through stimulation of macrophages. Nutrients 5(7): 2577-2589.

48. Thyer L, Ward E, Smith R, Branca JJ, Morucci G, et al. (2013) GC protein-derived macrophage-activating factor decreases $\alpha-\mathrm{N}$ acetylgalactosaminidase levels in advanced cancer patients. Oncoimmunology 2(8): e25769.

49. Bradstreet JJ, Vogelaar E, Thyer L (2012) Initial observations of elevated alpha-Nacetylgalactosaminidase activity associated with autism and observed reductions from Gc protein-macropahe activating factor injections. Autism Insights 4: 31.

50. Yamamoto N, Ushijima N, Koga Y (2009) Immunotherapy of HIV-infected patients with Gc protein-derived macrophage activating factor (GcMAF). J Med Virol 81(1):16-26.

51. Cecchi F, Pacini S, Gulisano M (2008) Heparin/heparan sulfate anticoagulant glycosaminoglycans in human plasma of healthy donors: preliminary study on a small group of recruits. Blood Coagul Fibrinolysis 19(5): 349-54.

52. Lu H, McDowell LM, Studelska DR, Zhang L (2010) Glycosaminoglycans in human and bovine serum: detection of twenty-four heparan sulfate and chondroitin sulfate motifs including a novel sialic acid-modified chondroitin sulfate linkage hexasaccharide. Glycobiol Insights 2010(2): 13-28.

53. Coppa GV, Gabrielli O, Bertino E (2013) Human milk glycosaminoglycans: the state of the art and future perspectives. Ital J Pediatr 39: 2.

54. Naik RJ, Sharma R, Nisakar D, Purohit G, Ganguli M (2015) Exogenous chondroitin sulfate glycosaminoglycan associate with arginine-rich peptide-DNA complexes to alter their intracellular processing and gene delivery efficiency. Biochim Biophys Acta 1848(4): 1053-1064.

55. DiMartino SJ, Kew RR (1999) Initial characterization of the vitamin D binding protein (Gc-globulin) binding site on the neutrophil plasma membrane: evidence for a chondroitin sulfate proteoglycan. J Immunol 163(4): 2135-2142.

56. Oesser S, Adam M, Babel W (1999) Oral administration of (14)C labelled gelatin hydrolysate leads to an accumulation of radioactivity in cartilage of mice (C57/BL. J Nutr 129(10), 1891-1895.

57. Wrenshall LE, Stevens RB, Cerra FB, Platt JL (1999) Modulation of macrophage and B cell function by glycosaminoglycans. J Leukoc Biol 66(3):391-400.

58. Ruggiero M, Ward E, Smith R (2014) Oleic Acid, deglycosylated vitamin D-binding protein, nitric oxide: a molecular triad made lethal to cancer. Anticancer Res 34(7): 3569-3578.

59. Vallières M, du Souich $P$ (2010) Modulation of inflammation by chondroitin sulfate. Osteoarthritis Cartilage 18(suppl 1): S1-S6.

60. http://www.mayoclinic.org/drugs-supplements/chondroitin-sulfate/ evidence/hrb-20058926.

61. Branca JJV, Smith RJ, Ward E, Ruggiero M (2015) Effect of glycosylated vitamin $\mathrm{D}$ binding protein complexed with oleic acid on human myeloma and Hodgkin's lymphoma cultures. Integr Cancer Sci Therap.

62. Menende, JA, Lupu R (2006) Mediterranean Dietary Traditions for the Molecular Treatment of Human Cancer: Anti-Oncogenic Actions of the Main Olive Oils Monounsaturated Fatty Acid Oleic Acid (18:1n-9). Curr Pharm Biotechnol 7(6): 495-502.

63. Williams MH, Van Alstyne EL, Galbraith RM (1988) Evidence of a novel association of unsaturated fatty acids with Gc (vitamin D-binding protein). Biochem Biophys Res Commun 153(3): 1019-1024.

64. Nakamura T, Aizawa T, Kariya R, Okada S, Demura M, et al. (2013) Molecular mechanisms of the cytotoxicity of human $\alpha$-lactalbumin made lethal to tumor cells (HAMLET) and other protein-oleic acid complexes. J Biol Chem 288(20): 14408-14416.

65. Nemashkalova EL (2013) Structural characterization of more potent alternatives to HAMLET, a tumoricidal complex of $\alpha$-lactalbumin and oleic acid. Biochemistry 52(36): 6286-6299.

66. Ruggiero M, Ward E, Smith R (2014) Oleic Acid, deglycosylated vitamin D-binding protein, nitric oxide: a molecular triad made lethal to cancer. Anticancer Res 34(7): 3569-3578.

67. Fang B (2013) Bovine lactoferrin binds oleic acid to form an anti-tumor complex similar to HAMLET. Biochim Biophys Acta 1841(4): 535-543.

68. Chin SF, Liu W, Storkson JM, Ha YL, Pariza MW (1992) Dietary sources of conjugated dienoic isomers of linoleic acid, a newly recognized class of anticarcinogens. Journal of Food Composition and Analysis 5(3): 185197. 
69. Belury MA, Vanden Heuvel JP (1997) Protection against cancer and heart disease by CLA: potential mechanisms of action. Nutr. Disease Update 1: 58-63.

70. Belury MA (2002) Dietary conjugated linoleic acid in health: physiological effects and mechanisms of action. Annu Rev Nutr 22: 505-531.

71. Scimeca JA (1999) Cancer inhibition in animals. In: Advances in Conjugated Linoleic Acid Research. In: Yurawecz MP, Mossobo MM, Kramer JKG, Pariza MW, Nelson GJ, (eds), AOCS Press 1: 420-443.
72. Inui T, Kubo K, Kuchiike D, Uto Y, Nishikata T, et al. (2015) Oral Colostrum Macrophage-activating Factor for Serious Infection and Chronic Fatigue Syndrome: Three Case Reports. ANTICANCER RESEARCH 35: 45454550 .

73. Uto Y, Kawai T, Sasaki T (2015) Degalactosylated/Desialylated Bovine Colostrum Induces Macrophage Phagocytic Activity Independently of Inflammatory Cytokine Production. Anticancer Res 35(8): 4487-4492. 